\title{
Automatic selection of initial points for exploratory vessel tracing in fluoroscopic images
}

\begin{abstract}
Automatic extraction of vessel centerlines has been an essential process in most of the image guided diagnosis and therapy applications. Among a considerable number of methods, direct exploratory tracing method is known to be an efficient solution for reliable extraction of vessel features from two-dimensional fluoroscopic images. The first step of most automatic exploratory tracing algorithms is collecting a number of candidate initial seed points and their initial tracing directions. To detect reliable initial points, a validation step is required to filter out the false candidates and avoid unnecessary tracing. Staring from reliable initial points, the algorithm efficiently extracts the centerline points along the initial direction until certain predefined criteria are satisfied. However, most of these algorithms suffer from incomplete results due to inappropriate selection of the initial seed points. The conventional seed point selection algorithms either rely merely on signal-to-noise ratio analysis, which results in a large number of false traces, or impose a set of strict geometrical validation rules that lead to more false negatives and require more computation time. This paper presents a new method for efficient selection of initial points for exploratory tracing algorithms. The proposed method improves the performance upon existing methods by employing a combination of geometrical and intensity-based approaches.
\end{abstract}

Keyword: Centerline extraction; Coronaries; Exploratory tracing; Seed point detection; Tracking 